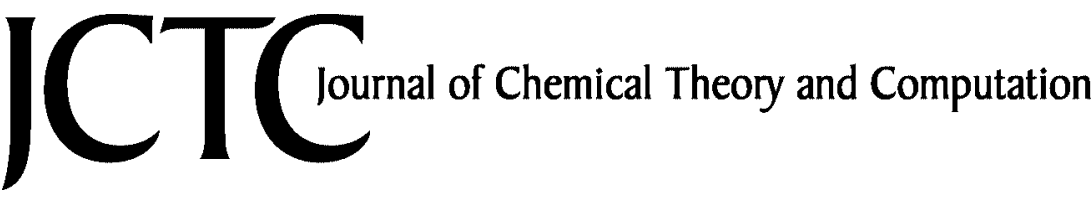

\section{Assessment of a Middle-Range Hybrid Functional}

\author{
Thomas M. Henderson, ${ }^{\dagger}$ Artur F. Izmaylov, ${ }^{\dagger}$ Gustavo E. Scuseria, ${ }^{* \dagger}$ and \\ Andreas Savin \\ Department of Chemistry, Rice University, 6100 Main Street, Houston, \\ Texas 77005-1892, and Laboratoire de Chimie Théorique, CNRS, Université Pierre et \\ Marie Curie, 4 Place Jussieu, F-75252 Paris, France
}

Received May 6, 2008

\begin{abstract}
While hybrid functionals are largely responsible for the utility of modern Kohn-Sham density functional theory, they are not without their weaknesses. In the solid state, the slow decay of their nonlocal Hartree-Fock-type exchange makes hybrids computationally demanding and can introduce unphysical effects. Both problems can be remedied by a screened hybrid which uses exact exchange only at short-range. Many molecular properties, in contrast, benefit from the inclusion of long-range exact exchange. Recently, the authors reconciled these two seemingly contradictory requirements by introducing the HISS functional [J. Chem. Phys. 2007, 127, 221103], which uses exact exchange only in the middle range. In this paper, we expand upon our previous work, benchmarking the performance of the HISS functional for several simple properties and applying it to the dissociation of homonuclear diatomic cations and to the polarizability of linear $\mathrm{H}_{2}$ chains to determine the importance of middle-range exact exchange for these systems, which are expected to be sensitive to the asymptotic exchange potential.
\end{abstract}

\section{Introduction}

Over the past several years, the Kohn-Sham (KS) construction in density functional theory ${ }^{1,2}$ (DFT) has become the dominant technique for predictions of the electronic structure of molecules and solids. ${ }^{3}$ This success is due to the method's combination of reasonable accuracy and low computational cost. The accuracy of a given KS calculation is largely controlled by the choice of the exchange-correlation functional, $E_{\mathrm{xc}}[n]$, which is known to be a functional of the density but whose precise form is unknown except in certain limiting cases.

The simplest class of exchange-correlation functionals are termed semilocal because they approximate the exchangecorrelation energy density at a point $\mathbf{r}$ as a function of the density at $\mathbf{r}$ and, possibly, its derivatives (which serve to incorporate information also in the neighborhood of the point r). Typical examples include the local density approximation (LDA), generalized gradient approximations (GGAs) such as the GGA of Perdew, Burke, and Ernzerhof (PBE), ${ }^{4}$ and

\footnotetext{
* Corresponding author. E-mail: guscus@ rice.edu.

†ice University.

¥ Université Pierre et Marie Curie.
}

meta-GGAs such as the functional of Tao, Perdew, Staroverov, and Scuseria (TPSS). ${ }^{5}$ Semilocal functionals are often derived from first principles without resort to empirical parameters, but unfortunately, they perform relatively poorly for many properties of interest. Therefore, it is common to use hybrid functionals, which mix a fraction of exact nonlocal Hartree-Fock-type exchange with the semilocal exchange provided by conventional functionals. ${ }^{6-10}$ Hybrid functionals tend to be significantly more accurate than their parent semilocal functionals, albeit at the cost of adding an extra parameter (the fraction of Hartree-Fock-type exchange) which is usually determined empirically.

But hybrids, also, suffer from several deficiencies. In extended systems, nonlocal Hartree-Fock-type exchange is expensive to compute since the lattice summations required converge very slowly in semiconductors and metals due to the slow spatial decay of the one-particle density matrix. ${ }^{11}$ On the other hand, in finite systems the exchange potential of a semilocal functional decays too rapidly, leading to errors in properties which sample density tails, and hybrids only partially remedy this defect.

Both of these problems can be alleviated by the use of range-separated hybrids, ${ }^{12-15}$ which split the Coulomb 
operator into short-range (SR) and long-range (LR) pieces, typically as

$\frac{1}{r}=\underbrace{\frac{\operatorname{erfc}(\omega r)}{r}}_{\mathrm{SR}}+\underbrace{\frac{\operatorname{erf}(\omega r)}{r}}_{\mathrm{LR}}$,

and then mix in different fractions of nonlocal exchange for the different regions. The screened hybrid functional of Heyd, Scuseria, and Ernzerhof, ${ }^{16,18}$ (HSE) uses a fraction of exact exchange in the short-range but none in the long-range; in doing so, it improves the convergence of the lattice sums required to evaluate the nonlocal exchange potential, thereby improving computational efficiency for solids and large molecules. ${ }^{19}$ The long-range-corrected $\mathrm{LC}-\omega \mathrm{PBE}^{20}$ hybrid of Vydrov and Scuseria uses no exact exchange at shortrange, but treats the long-range exclusively with Hartree-Focktype exchange, thus yielding the right answer in density tails and improving accuracy for a host of properties.

Recently, we introduced the HISS functional ${ }^{21}$ which uses exact exchange in the middle range (MR) only, with the intent of providing a functional with the computational advantages of HSE but with much of the accuracy of LC$\omega$ PBE. Preliminary results suggest that the functional referred to as HISS-B in ref 21 and called simply HISS here provides a sort of "best of both worlds" approach in that it yields uniformly accurate results for total atomic energies, heats of formation, reaction barrier heights, and band gaps of solids. In contrast, while both HSE and LC- $\omega$ PBE perform well for atomic energies and for heats of formation, HSE performs poorly for reaction barriers and LC- $\omega \mathrm{PBE}$ performs quite badly for band gaps.

The present work aims to systematically investigate the performance of the HISS functional for a wide variety of properties, providing benchmark results so that we can further assess what the functional does right and why it does so. In section 2, we discuss the HISS functional, after which we elaborate on its performance for several properties in section 3. We conclude with section 4 .

\section{HISS Functional}

The HISS functional is a multi-range hybrid which partitions the Coulomb operator into three pieces rather than the two given in eq 1 . This is accomplished by writing

$\frac{1}{r}=\underbrace{\frac{\operatorname{erfc}\left(\omega_{\mathrm{SR}} r\right)}{r}}_{\mathrm{SR}}+\underbrace{\frac{\operatorname{erf}\left(\omega_{\mathrm{LR}} r\right)}{r}}_{\mathrm{LR}}+\underbrace{\frac{\operatorname{erfc}\left(\omega_{\mathrm{LR}} r\right)-\operatorname{erfc}\left(\omega_{\mathrm{SR}} r\right)}{r}}_{\mathrm{MR}}$

where $\omega_{\mathrm{SR}}$ and $\omega_{\mathrm{LR}}$ are the two parameters defining the ranges and here take numerical values of $0.84 a_{0}^{-1}$ and $0.20 a_{0}{ }^{-1}$, respectively. Given this partition, the exchangecorrelation energy within HISS is written as

$$
E_{\mathrm{xc}}^{\mathrm{HISS}}=E_{\mathrm{x}}^{\mathrm{SR}-\mathrm{PBE}}+\frac{2}{5} E_{\mathrm{x}}^{\mathrm{MR}-\mathrm{PBE}}+\frac{3}{5} E_{\mathrm{x}}^{\mathrm{MR}-\mathrm{HF}}+E_{\mathrm{x}}^{\mathrm{LR}-\mathrm{PBE}}+E_{\mathrm{c}}^{\mathrm{PBE}}
$$

where HF refers to Hartree-Fock-type exchange, PBE for exchange refers to the exchange functional built from the PBE model exchange hole, ${ }^{22}$ and PBE for correlation refers
Table 1. Mean Errors and Mean Absolute Errors per Electron $(\mathrm{mH})$ in Total Atomic Energies for the First Eighteen Atoms from a Variety of Functionals Based on the PBE GGA ${ }^{a}$

\begin{tabular}{lcccc}
\hline functional & ME & MAE & $\max +$ & $\max -$ \\
\hline HISS & 4.50 & 4.84 & $7.69(\mathrm{Be})$ & $-3.11(\mathrm{H})$ \\
HSE & 6.06 & 6.21 & $8.07(\mathrm{Ar})$ & $-1.33(\mathrm{H})$ \\
LC- $\omega$ PBE & 4.37 & 5.05 & $7.45(\mathrm{Ar})$ & $-6.09(\mathrm{H})$ \\
PBE & 8.55 & 8.55 & $11.60(\mathrm{Ar})$ & \\
PBEh & 6.98 & 7.10 & $9.15(\mathrm{Ar})$ & $-1.15(\mathrm{H})$
\end{tabular}

${ }^{a}$ Also shown are maximum positive and negative deviations and the atoms to which they correspond.

to the original functional of Perdew, Burke, and Ernzerhof. The various parameters $\left(\omega_{\mathrm{SR}}, \omega_{\mathrm{LR}}\right.$, and the fraction of middlerange exact exchange) were obtained by fitting to the (small) AE6 set of atomization energies ${ }^{23}$ and the BH6 set of barrier heights $^{23}$ while insisting that performance for band gaps in solids be reasonable. Note that, as with most hybrids, we use a generalized Kohn-Sham scheme ${ }^{24}$ in which we employ the nonlocal exchange potential.

\section{Benchmarks}

With the HISS functional defined, we are ready to begin its assessment. Our intent in this work is to more exhaustively examine the effects of middle-range exact exchange than was done in ref 21.

Throughout, we will compare with other functionals in the "PBE family", including PBE itself, the PBEh global hybrid with $25 \%$ exact exchange, as well as the screened HSE06 hybrid and the long-range-corrected LC- $\omega$ PBE hybrid. All calculations are performed self-consistently using a development version of the GAUSSIAN program. ${ }^{25}$ Except where mentioned otherwise, we use the $6-311++\mathrm{G}(3 \mathrm{df}, 3 \mathrm{pd})$ basis set.

3.1. Previous Results. For the sake of completeness, we begin by expanding upon the results of ref 21, which studied atomic total energies, thermochemistry, reaction barrier heights, and band gaps in solids. We will discuss each of these in turn.

3.1.1. Atomic Energies. One important requirement of any successful functional is the ability to properly describe atomic total energies. Atomic energies are particularly important in describing heats of formation, atomization energies, cohesive energies, and so on. ${ }^{26}$ To test our results for total atomic energies, we consider the first eighteen atoms (H-Ar). In Table 1, we show mean error (ME) and mean absolute error (MAE) per electron for a variety of functionals; reference data is taken from the work of Chakravorty et al. ${ }^{27}$ Errors are defined as the result from the functional minus the reference result; positive errors thus indicate that the functional gives a result too low in absolute value. We clearly see that HISS, while not parametrized to atomic energies, nevertheless reproduces them very well, with accuracy comparable to that of LC- $\omega \mathrm{PBE}$ and significantly better than that of PBE or PBEh. These results may partially explain the success of HISS for heats of formation, which we address next.

3.1.2. Heats of Formation. Performance for equilibrium thermochemistry has long been one of the main requirements 
Table 2. Mean Errors and Mean Absolute Errors $(\mathrm{kcal} / \mathrm{mol})$ in the $\mathrm{G} 2$ and G3 Sets of Heats of Formation from a Variety of Functionals Based on the PBE GGA ${ }^{a}$

\begin{tabular}{|c|c|c|c|c|c|c|c|c|}
\hline \multirow[b]{2}{*}{ functional } & \multicolumn{4}{|c|}{ G2 } & \multicolumn{4}{|c|}{ G3 } \\
\hline & ME & MAE & $\max +$ & $\max -$ & ME & MAE & $\max +$ & $\max -$ \\
\hline HISS & 3.12 & 4.39 & $26.1\left(\mathrm{O}_{3}\right)$ & $-9.4\left(\mathrm{C}_{2} \mathrm{~F}_{4}\right)$ & 2.46 & 4.34 & $26.1\left(\mathrm{O}_{3}\right)$ & $-10.6\left(\mathrm{C}_{10} \mathrm{H}_{8}\right)$ \\
\hline LC- $\omega$ PBE & -0.36 & 3.73 & $16.3\left(\mathrm{P}_{2}\right)$ & $-20.6\left(\mathrm{C}_{2} \mathrm{~F}_{4}\right)$ & -0.93 & 4.25 & $16.3\left(\mathrm{P}_{2}\right)$ & $-21.4\left(\mathrm{C}_{2} \mathrm{~F}_{6}\right)$ \\
\hline PBE & -16.07 & 16.87 & $10.8\left(\mathrm{Si}_{2} \mathrm{H}_{6}\right)$ & $-50.5\left(\mathrm{C}_{2} \mathrm{~F}_{4}\right)$ & -21.69 & 22.22 & $10.8\left(\mathrm{Si}_{2} \mathrm{H}_{6}\right)$ & $-79.7\left(\mathrm{C}_{10} \mathrm{H}_{8}\right)$ \\
\hline PBEh & -2.42 & 4.87 & $21.3\left(\mathrm{SiF}_{4}\right)$ & $-19.8\left(\mathrm{C}_{5} \mathrm{H}_{5} \mathrm{~N}\right)$ & -4.72 & 6.66 & $21.3\left(\mathrm{SiF}_{4}\right)$ & $-35.6\left(\mathrm{C}_{10} \mathrm{H}_{8}\right)$ \\
\hline
\end{tabular}

${ }^{a}$ Also shown are maximum positive and negative deviations and the systems to which they correspond.

Table 3. Mean Errors and Mean Absolute Errors $(\mathrm{kcal} / \mathrm{mol})$ in the HTBH38 and NHTBH38 Sets of Reaction Barrier Heights from a Variety of Functionals Based on the PBE GGA

\begin{tabular}{|c|c|c|c|c|c|c|c|c|c|c|}
\hline \multirow[b]{3}{*}{ functional } & & & \multicolumn{8}{|c|}{$\begin{array}{l}\text { non-hydrogen-transfer } \\
\text { reactions of the NHTBH38 set }\end{array}$} \\
\hline & \multicolumn{2}{|c|}{$\begin{array}{l}\text { HTBH38 hydrogen } \\
\text { transfer (38) }\end{array}$} & \multicolumn{2}{|c|}{$\begin{array}{l}\text { heavy-atom } \\
\text { transfer (12) }\end{array}$} & \multicolumn{2}{|c|}{$\begin{array}{c}\text { nucleophilic } \\
\text { substitution (16) }\end{array}$} & \multicolumn{2}{|c|}{$\begin{array}{l}\text { unimolecular and } \\
\text { association (10) }\end{array}$} & \multicolumn{2}{|c|}{$\begin{array}{c}\text { full } \\
\text { NHTBH38 (38) }\end{array}$} \\
\hline & ME & MAE & ME & MAE & ME & MAE & ME & MAE & ME & MAE \\
\hline HISS & -1.3 & 1.7 & -2.1 & 2.4 & 0.6 & 0.8 & 1.0 & 2.7 & -0.2 & 1.8 \\
\hline HSE & -4.6 & 4.6 & -7.4 & 7.4 & -2.3 & 2.4 & -0.9 & 2.2 & -3.5 & 3.9 \\
\hline LC- $\omega$ PBE & -0.5 & 1.3 & -0.6 & 1.9 & 2.8 & 2.8 & 1.4 & 2.3 & 1.4 & 2.4 \\
\hline PBE & -9.7 & 9.7 & -15.3 & 15.3 & -6.8 & 6.8 & -3.1 & 3.5 & -8.5 & 8.6 \\
\hline PBEh & -4.6 & 4.6 & -7.0 & 7.0 & -1.7 & 1.9 & -0.8 & 2.3 & -3.1 & 3.6 \\
\hline
\end{tabular}

for methods in quantum chemistry. To assess this performance, we calculate heats of formation $\left(\Delta_{\mathrm{f}} H_{298^{\circ}}\right)$ in two standard benchmark test sets, namely the G2/97 set of 148 molecules, ${ }^{28}$ and the $\mathrm{G} 3 / 99$ set of 223 molecules; ${ }^{29} \mathrm{G} 3 / 99$ is a superset of G2/97 which primarily adds larger organic molecules.

In accordance with refs 28 and 30, we use equilibrium geometries and zero-point energies tabulated at the B3LYP/ 6-31G(2df,p) level of theory, with a frequency scale factor of 0.9854 . We calculate the total energies of the atoms and molecules in the test set with our chosen functional and basis set. Enthalpies of formation are then obtained by calculating atomization energies, adding tabulated thermal corrections and zero-point energies, and using the experimental enthalpies of formation of free atoms. Errors in $\Delta_{\mathrm{f}} H_{298^{\circ}}$ thus reflect errors in atomization energies. While this procedure does not produce a truly consistent atomization energy, since it does not use the molecular geometry predicted by the HISS functional, this is standard practice in benchmarking calculations (so as to separate errors in geometry from errors in energetics). Here and in what follows, we define errors as theory minus experiment.

Table 2 shows that HISS is as accurate as the best functionals we consider (and in ref 20, it was shown that LC- $\omega$ PBE delivers thermochemical performance competitive with heavily parametrized functionals). In particular, HISS clearly performs better than PBEh, and much better than PBE. Both of the latter are markedly less accurate for the G3 set than they are for the G2 set, which implies size-dependent errors for these functionals which are reduced significantly in HSE, LC- $\omega$ PBE, and HISS. We should point out that while the other functionals all tend to underestimate enthalpies of formation (i.e., the mean error is negative, albeit only slightly so in the case of LC- $\omega$ PBE), HISS tends to overestimate them instead. Calculations on the AE6 set of atomization energies show that using the HISS geometry instead of that specified by the benchmarking set decreases the MAE by $0.15 \mathrm{kcal} / \mathrm{mol}$.

3.1.3. Reaction Barrier Heights. Semilocal functionals generally underestimate reaction barriers, and often dramatically so. Transition states are not infrequently predicted to be lower in energy than the reactants or products. This error is connected to one-electron self-interaction error, which is particularly large for systems with stretched bonds which allow electrons to delocalize readily. Many semilocal functionals, in fact, deliver reasonable reaction barriers when the orbitals are taken from a self-interaction free method. ${ }^{31}$

A second, more consistent, route toward predicting reaction barrier heights with DFT is to use hybrid functionals with a large $(40-60 \%)$ fraction of nonlocal exchange. But while these functionals can accurately predict barrier heights, they perform poorly for equilibrium thermochemistry. Relatively few functionals do well for both, and most of those are heavily parametrized. Long-range-corrected hybrids, however, are also accurate for both reaction barriers and equilibrium thermochemistry. Since HISS contains a significant fraction of exact exchange over the length scales relevant in transition states, one might expect HISS to be another functional that gives accurate barrier height as well as thermochemistry.

To assess performance for reaction barriers, we consider the HTBH38/04 set of forward and reverse barrier heights for 19 hydrogen transfer reactions, ${ }^{32}$ and the NHTBH38/04 set of forward and reverse barrier heights for 19 nonhydrogen-transfer reactions. ${ }^{33}$ Geometries of all species as well as the best estimates for the experimental barrier heights are taken from ref 33 .

As can be seen from Table 3, only HISS and LC- $\omega$ PBE describe hydrogen transfer or heavy atoms transfer barriers with satisfactory accuracy, while for these reactions PBEh and HSE are inadequate and PBE is clearly unacceptable. For nucleophilic substitution reactions, PBEh performs quite 
well and HSE is almost as good as LC- $\omega \mathrm{PBE}$ but HISS gives excellent results. Note that LC- $\omega$ PBE uniformly overestimates the barriers to nucleophilic substitution, while PBE underestimates them; apparently, LC- $\omega \mathrm{PBE}$ includes too much exact exchange for this type of reaction, and HISS, which generally uses less exact exchange than LC- $\omega$ PBE, happens to give the right answer. All of the functionals perform similarly for unimolecular and association reactions. To examine the effects of geometry on the reaction barriers, we optimize the geometries of the reactants, products, and transition states in the BH6 set using HISS, which decreases the MAE by $0.09 \mathrm{kcal} / \mathrm{mol}$. Generally, LC- $\omega$ PBE outperforms HISS for reaction barriers, but HISS performs quite adequately for all categories and, in particular, yields better answers than does HSE [Note that there is an error in Table 1 of ref 21; the number given here for the mean absolute error in the HSE calculations of barrier heights in the NHTBH38 set is correct]; as we shall see in the following section, the opposite conclusions can be draw for band gaps.

3.1.4. Band Gaps in Solids. Finally, we consider a small set of band gap calculations to assess the performance of the HISS functional in solids, examining $\mathrm{Si}, \mathrm{C}, \mathrm{SiC}, \mathrm{BN}$, and BP. As seen from Table 4, both PBEh and LC- $\omega$ PBE consistently overestimate band gaps, and in the case of LC$\omega \mathrm{PBE}$, disastrously so. While the magnitude of the overestimation is somewhat in question due to the limited data available, the fact that these functionals do generally overestimate the band gap is certainly true, especially given that Hartree-Fock theory does the same.

While both PBEh and LC- $\omega$ PBE perform poorly for band gaps, HISS is somewhat better behaved. It still overestimates band gaps consistently, indicating that the amount of exact exchange included is (on average) more than is required for reproducing experimental band gaps. In this respect, the HSE functional has close to the optimal amount of exact exchange. The existence and, more interestingly, approximate universality of this optimal amount of exact exchange between two limits (PBE and PBEh) has been rationalized recently by examining excitation energies from time-dependent density functional calculations with periodic boundary conditions. ${ }^{34}$

We have also calculated the band gap in $\mathrm{MgO}$ and $\mathrm{NaCl}$, which we have excluded from the statistics above. For wide band gap insulators, the optical and fundamental gaps differ significantly. We have reasons to believe that band gaps obtained as band energy differences in band structure calculations with screened functionals tend to approximate optical instead of fundamental gaps. ${ }^{37}$ Thus, we have excluded these systems from the statistics in Table 4, which is then limited to semiconductors. For the two ionic solids we have considered, HISS is in error by about $0.9 \mathrm{eV}$, again comparable to HSE (which underestimates the gaps by about $1 \mathrm{eV})$. Note, however, that in contrast to HSE, the HISS functional does not consistently underestimate the band gaps in these two systems, but rather underestimates the gap in $\mathrm{NaCl}$ and overestimates it in $\mathrm{MgO}$. Interestingly, the PBEh global hybrid performs better than either HISS or HSE for these two systems, yielding band gaps in error by $0.6 \mathrm{eV}$.
Table 4. Mean Errors and Mean Absolute Errors (eV) in the Band Gaps for $\mathrm{Si}, \mathrm{C}, \mathrm{SiC}, \mathrm{BN}$, and BP from a Variety of Functionals Based on the PBE GGA ${ }^{a}$

\begin{tabular}{lrcc}
\hline functional & ME & MAE & \multicolumn{1}{c}{$\max$} \\
\hline HISS & 0.38 & 0.38 & $0.63(\mathrm{C})$ \\
HSE & -0.09 & 0.09 & $-0.28(\mathrm{BP})$ \\
${\text { LC- } \omega \text { PBE }^{b}}_{\text {PBE }}^{3.81}$ & 3.81 & $4.11(\mathrm{CSi})$ \\
PBEh & -1.10 & 1.10 & $-1.70(\mathrm{BN})$ \\
& 0.57 & 0.57 & $0.73(\mathrm{Si})$
\end{tabular}

${ }^{a}$ All basis sets and geometries are given in ref 35 . For all functionals, the geometries have been optimized at the HSE/ $\mathrm{m}-6311 \mathrm{G}^{* *}$ level of theory. ${ }^{36}$ Also shown are maximum absolute deviation and the system to which it corresponds. ${ }^{b}$ Excluding BN and $\mathrm{C}$, calculations on which we were unable to converge.

Table 5. Mean Errors and Mean Absolute Errors in IPs and EAs $(\mathrm{eV})$ and in PAs $(\mathrm{kcal} / \mathrm{mol})$ from a Variety of Functionals Based on the PBE GGA ${ }^{a}$

\begin{tabular}{|c|c|c|c|c|}
\hline functional & ME & MAE & $\max +$ & $\max -$ \\
\hline \multicolumn{5}{|c|}{$\mathrm{IP}(\mathrm{eV})$} \\
\hline HISS & 0.006 & 0.188 & $2.01(\mathrm{CN})$ & $-0.49\left(\mathrm{~B}_{2} \mathrm{~F}_{4}\right)$ \\
\hline HSE & -0.068 & 0.201 & $1.60(\mathrm{CN})$ & $-0.68\left(\mathrm{~B}_{2} \mathrm{~F}_{4}\right)$ \\
\hline LC- $\omega$ PBE & 0.073 & 0.192 & $1.97(\mathrm{CN})$ & $-0.46\left(\mathrm{~B}_{2} \mathrm{~F}_{4}\right)$ \\
\hline PBE & -0.105 & 0.235 & $1.11(\mathrm{CN})$ & $-1.01\left(\mathrm{BF}_{3}\right)$ \\
\hline PBEh & -0.064 & 0.199 & $1.61(\mathrm{CN})$ & $-0.67\left(\mathrm{~B}_{2} \mathrm{~F}_{4}\right)$ \\
\hline \multicolumn{5}{|c|}{$\mathrm{EA}(\mathrm{eV})$} \\
\hline HISS & -0.047 & 0.211 & $1.34\left(\mathrm{C}_{2}\right)$ & $-0.42\left(\mathrm{CH}_{3}\right)$ \\
\hline HSE & -0.025 & 0.165 & $1.09\left(\mathrm{C}_{2}\right)$ & $-0.37\left(\mathrm{HO}_{2}\right)$ \\
\hline LC- $\omega$ PBE & 0.019 & 0.177 & $1.41\left(\mathrm{C}_{2}\right)$ & $-0.31\left(\mathrm{CH}_{3}\right)$ \\
\hline PBE & 0.061 & 0.118 & $0.78\left(\mathrm{C}_{2}\right)$ & $-0.29\left(\mathrm{NO}_{2}\right)$ \\
\hline PBEh & -0.027 & 0.165 & $1.09\left(\mathrm{C}_{2}\right)$ & $-0.39\left(\mathrm{HO}_{2}\right)$ \\
\hline \multicolumn{5}{|c|}{$\mathrm{PA}(\mathrm{kcal} / \mathrm{mol})$} \\
\hline HISS & 1.07 & 1.51 & $4.6\left(\mathrm{C}_{2} \mathrm{H}_{2}\right)$ & $-1.5\left(\mathrm{SiH}_{4}\right)$ \\
\hline HSE & 0.09 & 1.11 & $3.6\left(\mathrm{C}_{2} \mathrm{H}_{2}\right)$ & $-1.5\left(\mathrm{SiH}_{4}\right)$ \\
\hline LC- $\omega$ PBE & 0.86 & 1.42 & $4.7\left(\mathrm{C}_{2} \mathrm{H}_{2}\right)$ & $-1.8\left(\mathrm{SiH}_{4}\right)$ \\
\hline PBE & -0.82 & 1.60 & $2.4\left(\mathrm{C}_{2} \mathrm{H}_{2}\right)$ & $-3.6\left(\mathrm{PH}_{3}\right)$ \\
\hline PBEh & 0.18 & 1.14 & $3.9\left(\mathrm{C}_{2} \mathrm{H}_{2}\right)$ & $-1.7\left(\mathrm{SiH}_{4}\right)$ \\
\hline
\end{tabular}

${ }^{a}$ Also shown are maximum positive and negative deviations and the systems to which they correspond.

3.2. Ionization Potentials, Electron Affinities, and Proton Affinities. Thus far, we have considered performance only for charge-neutral species. We can also consider positively or negatively charged systems, however, which play important roles in chemistry. Further, they can pose interesting challenges for density functionals, since electron attachment energies tend to sample the long-range part of the density which is poorly described by semilocal functionals.

To assess the quality of the HISS functional for charged species, we examine the $\mathrm{G} 2$ ion test set, ${ }^{38}$ excluding $\mathrm{N}_{2}{ }^{+}$ and $\mathrm{H}_{2} \mathrm{~S}^{+}$which do not converge at the GGA level. ${ }^{38}$ The set thus includes 86 ionization potentials (IPs) and 58 electron affinities (EAs). We also consider the eight proton affinities (PAs) in the G3/99 test set. Results are reported in Table 5 and are calculated as the difference between the selfconsistent energies of the neutral and charged species (i.e., these are " $\triangle \mathrm{SCF}$ " calculations).

In general, HISS performs quite well for IPs, yielding results essentially equivalent to those from HSE, PBEh, or LC- $\omega$ PBE, while having a very low mean signed error. For EAs, however, HISS underperforms significantly. Indeed, the performance for electron affinities is almost opposite what one might expect, since PBE is quite accurate while 
Table 6. Mean Errors and Mean Absolute Errors in Equilibrium Bond Lengths $(\AA)$ from a Variety of Functionals Based on the PBE GGA ${ }^{a}$

\begin{tabular}{lcccc}
\hline functional & ME & MAE & $\max +$ & $\max -$ \\
\hline HISS & -0.0100 & 0.0146 & $0.073\left(\mathrm{Li}_{2}\right)$ & $-0.070\left(\mathrm{~F}_{2}{ }^{+}\right)$ \\
HSE & -0.0007 & 0.0090 & $0.057\left(\mathrm{Li}_{2}\right)$ & $-0.050\left(\mathrm{~F}_{2}^{+}\right)$ \\
LC- $\omega$ PBE & -0.0087 & 0.0139 & $0.044\left(\mathrm{Li}_{2}\right)$ & $-0.055\left(\mathrm{P}_{4}\right)$ \\
PBE & 0.0156 & 0.0160 & $0.055\left(\mathrm{Li}_{2}\right)$ & $-0.009\left(\mathrm{~F}_{2}^{+}\right)$ \\
PBEh & -0.0013 & 0.0091 & $0.055\left(\mathrm{Li}_{2}\right)$ & $-0.052\left(\mathrm{~F}_{2}^{+}\right)$ \\
\hline
\end{tabular}

${ }^{a}$ Also shown are maximum positive and negative deviations and the systems to which they correspond.

functionals with better long-range exchange potentials have much higher error. For proton affinities, HISS improves only slightly over PBE.

3.3. Bond Lengths and Vibrational Frequencies. Aside from energetic quantities, we wish to predict structural information and other properties as well. We test the performance of HISS for molecular geometries by considering a test set of equilibrium bond lengths $\left(r_{\mathrm{e}}\right)$ compiled in ref 39 . Since $\mathrm{Be}_{2}$ is bound primarily by van der Waals forces, we have excluded it, and the remaining 95 covalently bonded molecules form the T-95R set of bond lengths defined by Vydrov and Scuseria. ${ }^{20}$ Most molecules are diatomic, though several polyatomic molecules with sufficiently high symmetry that the geometry is determined entirely by one bond length are also included. We point out that although for each molecule in this test set we need optimize only a single geometrical parameter, we use analytic energy gradients to do so, and we are not limited to predicting geometries of simple systems.

As seen in Table 6, HISS is comparable in accuracy to LC- $\omega$ PBE and slightly outperforms PBE, though without the systematic bias toward stretched bonds that the latter functional exhibits. Both PBEh and HSE are significantly more accurate.

Once equilibrium molecular geometries are obtained-thus providing some assessment of the accuracy of the first derivatives of the potential energy surface with respect to nuclear displacements-it is natural to calculate vibrational frequencies, thereby assessing the second derivatives as well. To this end, we consider the T-82F test set of 82 diatomic molecules, ${ }^{40}$ whose experimental harmonic vibrational frequencies have been well studied; once again, we exclude $\mathrm{Be}_{2}$, leaving us with 81 diatomics. Considering the excellent performance of PBEh and HSE for molecular geometries, we might expect them to be similarly accurate for vibrational frequencies, while HISS and LC- $\omega$ PBE might be expected to suffer somewhat from their inclusion of a large portion of exact exchange (since Hartree-Fock is known to strongly overestimate vibrational frequencies). As seen in Table 7, this is indeed the case. While PBE tends to underestimate vibrational frequencies, hybrid functionals do the opposite, and as the amount of exact exchange included gets larger, so too do errors in vibrational frequencies. This points toward inadequacies in the PBE correlation functional as the likely source of error, and we suggest that, with an improved correlation functional, HISS and LC- $\omega$ PBE are quite likely to deliver improved accuracy for these properties.
Table 7. Mean Errors and Mean Absolute Errors $\left(\mathrm{cm}^{-1}\right)$ for Harmonic Vibrational Frequencies from a Variety of Functionals Based on the PBE GGA ${ }^{a}$

\begin{tabular}{lcccc}
\hline functional & ME & MAE & $\max +$ & $\max -$ \\
\hline HISS & 72.3 & 74.6 & $328.2\left(\mathrm{O}_{2}{ }^{+}\right)$ & $-20.7(\mathrm{AIF})$ \\
HSE & 31.9 & 42.4 & $234.0\left(\mathrm{O}_{2}{ }^{+}\right)$ & $-39.1(\mathrm{AlH})$ \\
LC- $\omega$ PBE & 55.1 & 62.3 & $237.4\left(\mathrm{~F}_{2}{ }^{+}\right)$ & $-87.1\left(\mathrm{HF}^{+}\right)$ \\
PBE & -33.1 & 41.5 & $75.9\left(\mathrm{~F}_{2}^{+}\right)$ & $-175.3\left(\mathrm{HF}^{+}\right)$ \\
PBEh & 34.7 & 43.8 & $236.3\left(\mathrm{O}_{2}^{+}\right)$ & $-36.2(\mathrm{AlH})$ \\
\hline
\end{tabular}

${ }^{a}$.Also shown are maximum positive and negative deviations and the systems to which they correspond.

Table 8. Mean Absolute Errors (eV) for 19 Valence and 20 Rydberg Excitation Energies for $\mathrm{CO}, \mathrm{N}_{2}$, and $\mathrm{H}_{2} \mathrm{CO}$ Molecules from a Variety of Functionals Based on the PBE $\mathrm{GGA}^{a}$

\begin{tabular}{lcc}
\hline functional & valence & Rydberg \\
\hline HISS & 0.45 & 0.18 \\
HSE & 0.40 & 0.79 \\
LC- $\omega$ PBE & 0.33 & 0.15 \\
PBE & 0.43 & 1.74 \\
PBEh & 0.39 & 0.85 \\
\hline
\end{tabular}

${ }^{a}$ The augmented Sadlej pVTZ basis is used. ${ }^{39}$

3.4. Electronic Excitation Energies. While the first several benchmarks have focused on equilibrium properties of molecules in their ground state, these can be predicted accurately by many semilocal functionals or global hybrids as well, and for these properties, the complexity of rangeseparated hybrids are not essential. More interesting is performance for properties sensitive to the exchange potential, and especially for those properties sensitive to the longrange exchange potential for which many functionals perform rather poorly.

We begin by considering electronic excitation spectra of three small molecules: $\mathrm{CO}, \mathrm{N}_{2}$, and $\mathrm{H}_{2} \mathrm{CO}$. Excitation spectra of these systems have been studied before, and in Table 8, we investigate the same 19 valence and 20 Rydberg excitations that have been assessed in ref 42 . All functionals give roughly similar performance for valence excitations, with errors on the order of $0.4 \mathrm{eV}$. This changes drastically for the Rydberg excitations, which are more sensitive to the long-range exchange-correlation potential and its derivative. Thus, it is interesting to note that although the exchange potential from HISS inherits the exponential decay of PBE rather than the $1 / r$ decay of LC- $\omega$ PBE, HISS nevertheless performs quite well for these Rydberg excitations. We speculate that these Rydberg excitations, which represent only the lower-lying members of the Rydberg series, are not high enough in energy to sample the truly long-range part of the potential. As we go further up in the Rydberg series, however, note that deficiencies in the basis set generally dominate other considerations.

3.5. Rydberg Excitation in the Hydrogen Atom. Let us return to the issue of Rydberg states in greater detail. Rydberg states are characterized by their progression toward an ionization threshold, with the $n^{\text {th }}$ Rydberg excitation energy given by

$$
\omega_{n}=\mathrm{IP}-\frac{1}{2(n-\delta)^{2}}
$$


Table 9. TDDFT Excitation Energies in the Hydrogen Atom from a Variety of Functionals Based on the PBE GGA Compared to the Exact Result ${ }^{a}$

\begin{tabular}{|c|c|c|c|c|c|c|c|}
\hline$n$ & exact & HF & LC- $\omega$ PBE & HISS & HSE & PBEh & PBE \\
\hline 2 & 10.2043 & 10.2043 & 9.1435 & 9.2510 & 8.5344 & 8.4411 & 7.5806 \\
\hline 3 & 12.0940 & 12.0939 & 10.7855 & 9.4311 & 8.7252 & 8.9546 & 7.5976 \\
\hline 4 & 12.7553 & 12.7553 & 11.3885 & 9.4394 & 8.7336 & 9.0478 & 7.6075 \\
\hline 6 & 13.2278 & 13.2278 & 11.8315 & 9.4847 & 8.7791 & 9.1001 & 7.6589 \\
\hline 7 & 13.3280 & 13.3280 & 11.9273 & 9.5347 & 8.8295 & 9.1234 & 7.7144 \\
\hline 8 & 13.3931 & 13.3931 & 11.9898 & 9.6206 & 8.9161 & 9.1753 & 7.8086 \\
\hline $\mathrm{IP}^{b}$ & 13.6057 & 13.6057 & 12.1972 & 9.4285 & 8.7226 & 9.1302 & 7.5944 \\
\hline $\mathrm{IP}^{c}$ & 13.6057 & 13.6057 & 13.7765 & 13.6953 & 13.6467 & 13.6417 & 13.6054 \\
\hline
\end{tabular}

${ }^{a}$ Also included are Hartree-Fock and TDHF, errors in which are purely due to the basis set. The last two rows contain the ionization potential as calculated in two different ways. All results are in electronvolts, using a conversion factor of $1 E_{\mathrm{h}}=27.2114 \mathrm{eV}$. ${ }^{b}$ lonization potential obtained as $-\varepsilon$ номо. ${ }^{c}$ Ionization potential obtained via $\triangle S C F$ between the neutral and the cation.

where $\delta$ is the quantum defect (and is zero in the hydrogen atom). We can thus, in principle, extract ionization potentials in at least three ways. First, we can directly evaluate the difference between the self-consistent energies of the charged and neutral species in a $\triangle \mathrm{SCF}$ calculation. Second, we can use the fact that, in the exact theory, the ionization potential is given by the opposite of the highest occupied orbital energy: ${ }^{1}$

$$
\mathrm{IP}=-\varepsilon_{\mathrm{HOMO}}
$$

Third, we can evaluate the limit of the Rydberg excitation spectra. All three methods should in principle give the same result but in practice give different answers due to deficiencies in the functionals and basis. Additionally, the quality of the Rydberg excitation spectra is generally rendered suspect for semilocal functionals since most calculations neglect both the current-dependence and the frequencydependence of the exchange-correlation kernel.

To examine the relation between the IP as calculated in these approaches, we wish to consider the hydrogen atom, in which there is no question that all excitations are Rydberg in character. Further, the $\triangle \mathrm{SCF}$ IP is simply the groundstate energy, so there is no question of error cancelation in evaluating it. Self-interaction error is a particularly large problem for one-electron systems, and all errors are in some way or another manifestations of it. We use an even-tempered expansion of 48 Gaussian functions with exponents between $10^{6}$ and $10^{-4}$, inclusive. Since all basis functions are spherical we use a one-point angular grid, and we use a 999point radial grid. Results are given in Table 9. As discussed above, we make the adiabatic approximation for the exchangecorrelation kernel and neglect any current dependence.

Let us begin with Hartree-Fock, which is of course exact within the limitations imposed by our finite basis set. The IP as calculated in a $\Delta-\mathrm{SCF}$ fashion is identical to that given by the occupied orbital energy, and within our basis, we describe the first nine Rydberg states $(n=2$ through $n=$ 10) or so almost exactly (error in the excitation energy for $n=10$ is only $0.4 \mathrm{meV}$ ). The Rydberg excitation energies approach the IP, and at $n=8$ are still about $0.2 \mathrm{eV}$ away, exactly as they should be.

Turning to LC- $\omega \mathrm{PBE}$, we find a slightly different story. The IP as calculated by $\triangle \mathrm{SCF}$ is $13.8 \mathrm{eV}$, with the error due primarily to the PBE correlation energy. But the IP as calculated by $-\varepsilon$ is only $12.2 \mathrm{eV}$; the difference reflects selfinteraction error. Past the first few excitations, there is an almost constant error of $1.4 \mathrm{eV}$ in the excitation energies; the LC- $\omega$ PBE Rydberg series is converging (as it should) to $-\varepsilon$. In other words, while having the right asymptotic potential is necessary, it is insufficient to guarantee that Rydberg excitations are properly described, and in longrange-corrected functionals, we also require that the orbital energy is correct.

Considering next PBE, the story changes again. The IP as calculated by $\triangle \mathrm{SCF}$ is $13.6 \mathrm{~V}$, indicating minimal selfinteraction error in the energy. On the other hand, the IP as calculated by $-\varepsilon$ is only $7.6 \mathrm{eV}$, indicating a very large selfinteraction error in the potential. The excitation energies calculated by TDDFT do not even form an obvious Rydberg series, and only one of them is below $-\varepsilon$. Several other excitations lie closely spaced but just above $-\varepsilon$. The fact that these states do not lie below $-\varepsilon$ may be due to inadequacies of our basis set.

The same qualitative story is true in HISS and HSE, which far from the nucleus resemble PBE. The PBEh global hybrid has several excitations below $-\varepsilon$, but it is not clear that they form a Rydberg series per se.

3.6. Dissociation of Homonuclear Diatomic Cations. The dissociation of homonuclear diatomic cations $\left(\mathrm{X}_{2}{ }^{+}\right)$has proven to be a challenge for density functional methods. Asymptotically, these systems can dissociate symmetrically to two fractionally charged species $\left(2 \mathrm{X}^{+1 / 2}\right)$ or asymmetrically to one neutral and one charged component $\left(\mathrm{X}+\mathrm{X}^{+}\right)$. In the exact theory, these limits must be degenerate. However, this is rarely the prediction of single determinant methods. Hartree-Fock theory predicts the symmetric dissociation limit to lie above the asymmetric limit-it frequently prefers to break symmetry and localize electrons. Semilocal functionals predict the opposite, and the potential energy curve goes through a maximum before turning over into a $1 /(4 R)$ decay far away as the two species interact Coulombically. Global hybrids like PBEh behave similarly, though the turnover is not as pronounced. The LC- $\omega$ PBE functional is somewhat better behaved-the potential energy curve does not have a transition state as in PBE, PBEh, and 

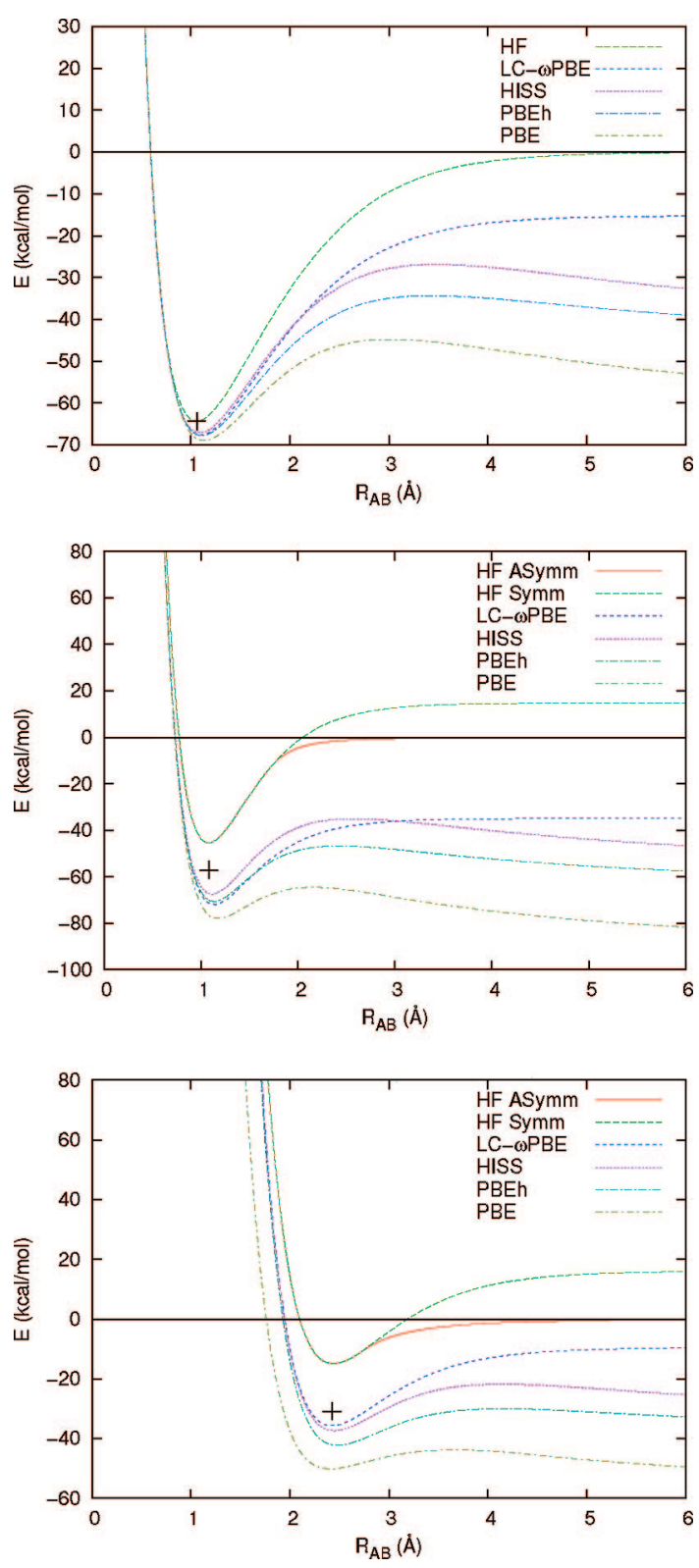

Figure 1. Dissociation of several homonuclear diatomic cations with HF theory and with several functionals based on the PBE GGA. The experimental equilibrium bond length and dissociation energy is marked by the symbol "+". (top panel) $\mathrm{H}_{2}{ }^{+}$(experiment, ref 41). (middle panel) $\mathrm{He}_{2}{ }^{+}$(experiment, ref 42). (bottom panel) $\mathrm{Ar}_{2}{ }^{+}$(experiment, ref 43).

HSE, but it predicts the symmetric dissociation limit to be lower in energy than the asymmetric limit.

We therefore examine the performance of the HISS functional for the dissociation of $\mathrm{H}_{2}{ }^{+}, \mathrm{He}_{2}{ }^{+}$, and $\mathrm{Ar}_{2}{ }^{+}$, with the potential energy curves given in Figure 1. The qualitative behavior of HISS is similar to that of PBEh and PBE, with an artificial transition state in the potential energy curve. However, HISS tends to push this point off to slightly larger internuclear separation. In other words, eventually the lack of long-range exact exchange causes HISS to break down and gives the type of result we expect to see for a semilocal functional, but this is delayed to longer bond lengths. Note that these calculations used Dunning's aug-cc-pVQZ basis set.

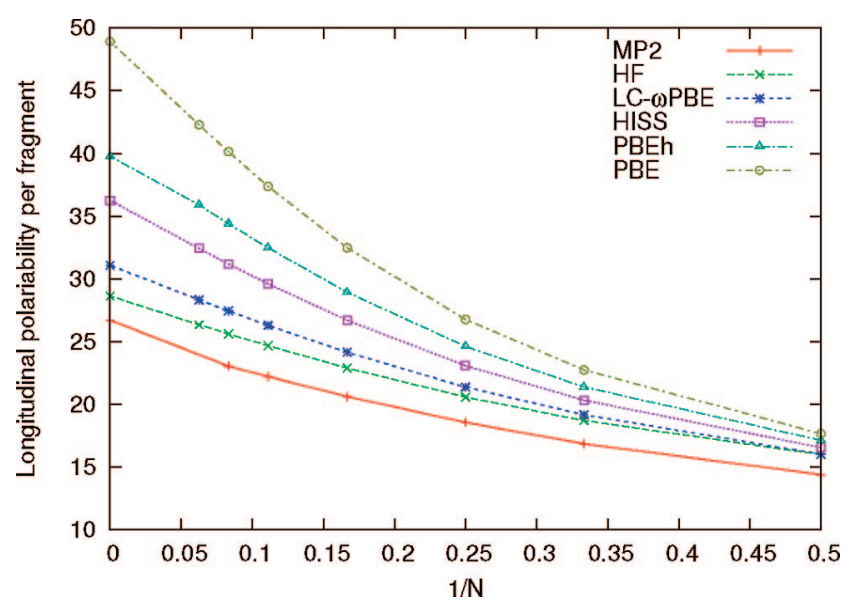

Figure 2. Longitudinal polarizability per $\mathrm{H}_{2}$ fragment $\left(a_{0}{ }^{3}\right)$ with $\mathrm{HF}, \mathrm{MP2}$, and several functionals based on the PBE GGA, plotted against one over the number of $\mathrm{H}_{2}$ fragments. The $\mathrm{PBC}$ limit is thus $1 / \mathrm{N} \rightarrow 0$.

3.7. Polarizabilities in $\mathbf{H}_{2}$ Chains. One well-known failure of semilocal functionals is in describing the polarizability of extended systems. Essentially, by artificially favoring electron delocalization due to self-interaction error, semilocal functionals tend to overestimate polarizabilities, particularly for large systems. This failure is largely corrected by long-range corrected exchange functionals. ${ }^{46,47} \mathrm{We}$ therefore wish to test the performance of HISS for this case, to determine whether the long-range-correction is critical or whether polarizabilities, like reaction barriers, are more sensitive to the presence of middle range exact exchange.

Our test systems constitute one-dimensional chains of hydrogen molecules aligned colinearly with interatomic and intermolecular distances of 2 and 3 au, respectively. As shown in ref 48 , accurately predicting the polarizability of the $\mathrm{H}_{2}$ linear chain is particularly difficult for semilocal functionals. Figure 2 presents the longitudinal polarizability per $\mathrm{H}_{2}$ fragment for oligomers of various lengths and the periodic chain. All periodic estimates except that for MP2 have been obtained by using our analytic polarizability code with periodic boundary conditions. ${ }^{47}$ For the periodic MP2 polarizabilities, we used finite electric field calculations ${ }^{50}$ of MP2 unit cell energies. ${ }^{51}$ All calculations used the $6-311 \mathrm{G}^{* *}$ basis set.

The polarizabilities obtained correlate with the amount of exact exchange in a straightforward way, with HISS yielding results intermediate between LC- $\omega \mathrm{PBE}$ and PBEh, in agreement with the qualitative results for homonuclear diatomic cations. Apparently, the amount of exact exchange is more important for this property than the range in which it is included. This is in agreement with self-interaction-corrected calculations of the same system, ${ }^{52}$ which show that as the self-interaction error decreases, so does the error in the polarizability.

\section{Conclusions}

Range-separated exchange functionals have much to offer over conventional hybrids, allowing for simple functionals which can do many things right. A screened hybrid which 
includes exact exchange only at short-range can provide accuracy essentially equivalent to that of the parent hybrid while increasing computational efficiency in solids and large molecules. A long-range-corrected hybrid which includes exact exchange only at long-range can simultaneously describe both thermochemistry and kinetics, as well as a wide variety of problems sensitive to the asymptotic form of the exchange potential.

By compromising between these two approaches, the HISS functional can accurately and efficiently predict atomization energies, barrier heights, and band gaps in solids. For properties sensitive to the longer-range exchange potential, HISS yields results, as one might expect, intermediate between those from the PBEh global hybrid and the LC$\omega \mathrm{PBE}$ long-range-corrected hybrid. In the cases of the dissociation curves of homonuclear diatomic cations and the polarizabilities of $\mathrm{H}_{2}$ chains, for which both semilocal and global hybrids fail, HISS is reasonably accurate and begins to fail only as the system gets large or spreads far apart. The same general observation holds for electronically excited states: HISS describes low-lying Rydberg states very well, presumably because these states are not too diffuse, and it is only as the states get higher in energy and more spread out that HISS will break down even if the basis set is otherwise capable of describing these excitations.

Of course, middle-range exact exchange is not a panacea, and for some properties, HISS performs no better than, or sometimes worse than, a global hybrid. As a rough rule of thumb, HISS underperforms in many of the same situations as does LC- $\omega$ PBE. This need not be too surprising, as both functionals use a large amount of exact exchange but use the standard PBE correlation functional. With their large fraction of exact exchange, neither HISS nor LC- $\omega$ PBE can be expected to benefit from the full error cancelation in semilocal functionals. By refining the correlation functional used with range-separated hybrids, performance for problematic cases should be improved further. Local rangeseparated exchange hybrids, which use make the fraction of exact exchange at each range a function of space, are also a route toward improved functionals. ${ }^{53-56}$

Acknowledgment. A.F.I. would like to thank Vincenzo Russo for help with the TDDFT assessment. This work was supported by the National Science Foundation (CHE0457030), the Department of Energy (DE-FG02-04ER15523), the Welch Foundation (C-0036), and ARO-MURI DAAD19-03-1-0169.

Supporting Information Available: Details of the basis sets used for our calculations in extended systems. This material is available free of charge via the Internet at http:// pubs.acs.org.

\section{References}

(1) Parr, R. G.; Yang, W. Density Functional Theory of Atoms and Molecules; Oxford University Press: New York, 1989.

(2) Dreizler, R. M.; Gross, E. K. U. Density Functional Theory; Plenum Press: New York, 1995.

(3) Scuseria, G. E.; Staroverov, V. N. Progress in the development of exchange-correlation functionals. In Theory and Applications of Computational Chemistry: The First 40 Years; Dykstra, C. E., Frenking, G., Kim, K. S., Scuseria, G. E., Eds.; Elsevier: Amsterdam, The Netherlands, 2005; pp 669-724.
(4) Perdew, J. P.; Burke, K.; Ernzerhof, M. Phys. Rev. Lett. 1997, 78, 1396.

(5) Tao, J.; Perdew, J. P.; Staroverov, V. N.; Scuseria, G. E. Phys. Rev. Lett. 2003, 91, 146401.

(6) Becke, A. D. J. Chem. Phys. 1993, 98, 1372.

(7) Becke, A. D. J. Chem. Phys. 1993, 98, 5648.

(8) Stephens, P. J.; Devlin, F. J.; Chabalowski, C. F.; Frisch, M. J. J. Phys. Chem. 1994, 98, 11623.

(9) Ernzerhof, M.; Scuseria, G. E. J. Chem. Phys. 1999, 110, 5029.

(10) Adamo, C.; Barone, V. J. Chem. Phys. 1999, 110, 6158.

(11) Monkhorst, H. J. Phys. Rev. B 1979, 20, 1504.

(12) Savin, A. On degeneracy, near-degenaracy and density functional theory. In Recent Developments and Applications of Modern Density Functional Theory; Seminario, J. M., Ed.; Elsevier: Amsterdam, The Netherlands, 1996; p 327.

(13) Leininger, T.; Stoll, H.; Werner, H.-J.; Savin, A. Chem. Phys. Lett. 1997, 275, 151.

(14) Gerber, I. C.; Angyan, J. G. Chem. Phys. Lett. 2005, 415, 100.

(15) Gerber, I. C.; Angyan, J. G.; Marsman, M.; Kresse, G. J. Chem. Phys. 2007, 127, 054101.

(16) Heyd, J.; Scuseria, G. E.; Ernzerhof, M. J. Chem. Phys. 2003, $118,8207$.

(17) Heyd, J.; Scuseria, G. E. J. Chem. Phys. 2004, 120, 7274.

(18) Heyd, J.; Scuseria, G. E. J. Chem. Phys. 2004, 121, 1187.

(19) Izmaylov, A. F.; Scuseria, G. E.; Frisch, M. J. J. Chem. Phys. 2006, 125, 104103.

(20) Vydrov, O. A.; Scuseria, G. E. J. Chem. Phys. 2006, 125, 234109.

(21) Henderson, T. M.; Izmaylov, A. F.; Scuseria, G. E.; Savin, A. J. Chem. Phys. 2007, 127, 221103.

(22) Ernzerhof, M.; Perdew, J. P. J. Chem. Phys. 1998, 109, 3313.

(23) Lynch, B. J.; Truhlar, D. G. J. Phys. Chem. A 2003, 107, 8996.

(24) Seidl, A.; Görling, A.; Vogl, P.; Majewski, J. A.; Levy, M. Phys. Rev. B 1996, 53, 3764.

(25) Frisch, M. J. ; Trucks, G. W.; Schlegel, H. B.; Scuseria, G. E.; Robb, M. A. ; Cheeseman, J. R. ; Montgomery, J. A., Jr.; Vreven, T.; Scalmani, G.; Kudin, K. N.; Iyengar, S. S. ; Tomasi, J. ; Barone, V. ; Mennucci, B.; Cossi, M.; Rega, N. ; Petersson, G. A. ; Nakatsuji, H. ; Hada, M. ; Ehara, M.; Toyota, K.; Fukuda, R. ; Hasegawa, J. ; Ishida, M. ; Nakajima, T. ; Honda, Y. ; Kitao, O. ; Nakai, H.; Li, X.; Hratchian, H. P.; Peralta, J. E. ; Izmaylov, A. F.; Brothers, E.; Staroverov, V.; Kobayashi, R. ; Normand, J. ; Burant, J. C.; Millam, J. M.; Klene, M. J.; Knox, E.; Cross, J. B.; Bakken, V.; Adamo, C.; Jaramillo, J.; Gomperts, R.; Stratmann, R. E.; Yazyev, O.; Austin, A. J.; Cammi, R.; Pomelli, C.; Ochterski, J. W.; Ayala, P. Y.; Morokuma, K.; Voth, G. A.; Salvador, P.; Dannenberg, J. J.; Zakrzewski, V. G.; Dapprich, S.; Daniels, A. D.; Strain, M. C.; Farkas, O.; Malick, D. K.; Rabuck, A. D.; Raghavachari, K.; Foresman, J. B.; Ortiz, J. V.; Cui, Q.; Baboul, A. G.; Clifford, S.; Cioslowski, J.; Stefanov, B. B.; Liu, G.; Liashenko, A.; Piskorz, P.; Komaromi, I.; Martin, R. L.; Fox, D. J.; Keith, T.; Al-Laham, M. A.; Peng, C. Y.; Nanayakkara, A.; Challacombe, M.; Chen, W.; Wong, M. W.; Pople, J. A. 
Gaussian Development Version, revision E.05; Gaussian, Inc.: Wallingford CT, 2006.

(26) Brothers, E. N.; Scuseria, G. E. J. Chem. Theory Comput. 2006, 4, 1045.

(27) Chakravorty, S. J.; Gwaltney, S. R.; Davidson, E. R.; Parpia, F. A.; Fischer, C. F. Phys. Rev. A 1993, 47, 3649.

(28) Curtiss, L. A.; Raghavachari, K.; Redfern, P. C.; Pople, J. A. J. Chem. Phys. 1997, 106, 1063.

(29) Curtiss, L. A.; Raghavachari, K.; Redfern, P. C.; Pople, J. A. J. Chem. Phys. 2000, 112, 7374.

(30) Curtiss, L. A.; Redfern, P. C.; Raghavachari, K.; Pople, J. A. J. Chem. Phys. 2001, 114, 108.

(31) Janesko, B. G.; Scuseria, G. E. J. Chem. Phys., in press.

(32) Zhao, Y.; Lynch, B. J.; Truhlar, D. G. Phys. Chem. Chem. Phys. 2004, 7, 43.

(33) Zhao, Y.; González-García, N.; Truhlar, D. G. J. Phys. Chem. A 2006, 110, 4942.

(34) Brothers, E. N.; Izmaylov, A. F.; Normand, J. O.; Barone, V.; Scuseria, G. E. J. Chem. Phys. 2008, 129, 011102.

(35) See the Supporting Information.

(36) Heyd, J.; Peralta, J. E.; Scuseria, G. E.; Martin, R. L. J. Chem. Phys. 2005, 123, 174101.

(37) Izmaylov, A. F.; Scuseria, G. E. Why are TD-DFT excitations in solids equal to band structure energy gaps for semilocal functionals and how does nonlocal Hartree-Fock type exchange introduce excitonic effects? J. Chem. Phys. 2008, 129, 034101 .

(38) Curtiss, L. A.; Redfern, P. C.; Raghavachari, K.; Pople, J. A. J. Chem. Phys. 1998, 109, 42.

(39) Staroverov, V. N.; Scuseria, G. E.; Tao, J.; Perdew, J. P. J. Chem. Phys. 2004, 121, 11507.
(40) Staroverov, V. N.; Scuseria, G. E.; Tao, J.; Perdew, J. P. J. Chem. Phys. 2003, 119, 12129.

(41) Sadlej, A. J. Theor. Chim. Acta 1991, 79, 123.

(42) Zhao, Y.; Truhlar, D. G. J. Phys. Chem. A 2006, 110, 13126.

(43) Schaad, L. J.; Hicks, W. V. J. Chem. Phys. 1970, 53, 851.

(44) Xie, J.; Poirier, B.; Gellene, G. I. J. Chem. Phys. 2005, 122, 184310 .

(45) Wüest, A.; Merkt, F. J. Chem. Phys. 2004, 210, 638.

(46) Iikura, H.; Tsuneda, T.; Yanai, T.; Hirao, K. J. Chem. Phys. 2001, 115, 3540.

(47) Sekino, H.; Maeda, Y.; Kamiya, M. Mol. Phys. 2005, 103, 2183.

(48) van Faassen, M.; de Boeij, P. L.; van Leeuwen, R.; Berger, J. A.; Snijders, J. G. Phys. Rev. Lett. 2002, 88, 186401.

(49) Izmaylov, A. F.; Brothers, E. N.; Scuseria, G. E. J. Chem. Phys. 2006, 125, 224105.

(50) Kudin, K. N.; Scuseria, G. E. J. Chem. Phys. 2000, 113, 7779.

(51) Ayala, P. Y.; Kudin, K. N.; Scuseria, G. J. Chem. Phys. 2001, $115,9698$.

(52) Ruzsinksky, A.; Perdew, J. P.; Csonka, G. I.; Scuseria, G. E.; Vydrov, O. A. Phys. Rev. A, in press.

(53) Jaramillo, J.; Scuseria, G. E.; Ernzerhof, M. J. Chem. Phys. 2003, 118, 1068.

(54) Janesko, B. G.; Scuseria, G. E. J. Chem. Phys. 2007, 127, 164117.

(55) Arbuznikov, A. V.; Kaupp, M. J. Chem. Phys. 2007, 127, 194102.

(56) Janesko, B. G.; Scuseria, G. E. J. Chem. Phys. 2008, 128, 084111.

CT800149Y 